\title{
Structure and Mechanical Properties of (CrAl)N and (CrAISiY)N Coatings Prepared by Asymmetrical Bipolar DC Magnetron Sputtering
}

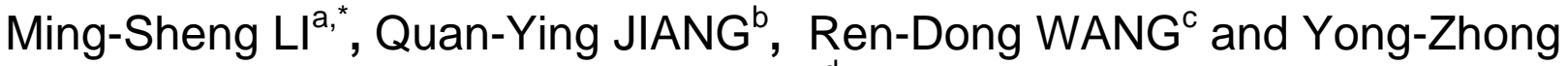 \\ FAN $^{\mathrm{d}}$
}

Jiangxi Normal University of Science and Technology, Nanchang, 330013, China

amshli@163.com, bqyjiang0627@163.com, c1023311032@qq.com, dyzfanbut@qq.com

Keywords: Arc ion plating, (CrAl)N, (CrAISiY)N, Micro structure, Hardness, Combination strength

\begin{abstract}
Composite (CrAl)N and (CrAlSiY)N hard coatings were deposited on 1Cr11Ni2W2MoV steel substrates by arc ion plating system under negative bias of $0 \mathrm{~V}, 100 \mathrm{~V}$ and $200 \mathrm{~V}$ respectively. Pulsed bias gave rise to the deviation of elements, the decreased deposition rate, and the change of the preferred orientation, the crystalline refining, the dense structure and improved hardness. The doping with small amount of Si and $\mathrm{Y}$ did not change the phase structure, but (CrAlSiY)N coatings possessed higher hardness value than $(\mathrm{CrAl}) \mathrm{N}$ coatings in case of the same deposition parameters. The harder coatings exhibited the lower toughness and the lower scratch critical load (Lc).
\end{abstract}

\section{Introduction}

CrN has been widely utilized as protective hard coatings for cutting and forming tools due to its high hardness (15 20 GPa) and good chemical stability [1, 2]. The performance of CrN could be improved significantly by the introduction of elements $\mathrm{Al}$ to form ternary (CrAl)N of B1 phase [3, 4]. CrAlN coatings possess higher hardness and oxidation-resistance compared with $\mathrm{CrN}$ coatings. The spinodal phase segregation of $\mathrm{B} 1-(\mathrm{CrAl}) \mathrm{N}$ does not take place even at more than $900{ }^{\circ} \mathrm{C}$ [5]. And since both $\mathrm{Al}$ and $\mathrm{Cr}$ could form protective oxides at high temperature, (CrAl)N coatings exhibit excellent oxidation resistance.

The alloying of (CrAl)N with silicon to form quaternary (CrAlSi)N could lead to crystalline refinement, super-hardness and improved anti-oxidation properties [6, 7]. Besides, there are reports to describe that $\mathrm{Y}$ in (CrAlY)N coatings segregates at the grain boundaries during oxidation and blocks grain boundary out-diffusion of metal and in-diffusion of oxygen ions and thus causes improved oxidation resistance [8, 9]. Therefore, the simultaneous introduction of Si and Y into the $(\mathrm{CrAl}) \mathrm{N}$ coatings is expected to further improve oxidation-resistance of the coatings.

The most common way to deposit $(\mathrm{CrAl}) \mathrm{N}$ coatings is to use a $\mathrm{CrAl}$ target material with corresponding composition [9]. But it is difficult to produce $\mathrm{CrAl}$ alloy target with a high $\mathrm{Al}$ ratio owing to the formation of brittle intermetallic compound phases (IMCs) [10]. An alternative method is to use separate elemental $\mathrm{Cr}$ and $\mathrm{Al}$ sources to and adjust sputtering power of the two targets individually [11]. In this case bipolar pulse DC magnetron sputtering (BPDMS) is the ideal technique for deposition of CrAlN in order to avoid "anode disappearing" and "arcing" phenomenon owing to the formation of insulating AlN on the surface of the Al target and the deposition of AlN on the inner surface of the deposition chamber [11].

\section{Experimental Methods}

Wrought martensite $1 \mathrm{Cr} 11 \mathrm{Ni} 2 \mathrm{~W} 2 \mathrm{MoV}$ steel sheets $(15 \mathrm{~mm} \times 10 \mathrm{~mm} \times 2 \mathrm{~mm})$ and tungsten carbide rods ( $\Phi 10 \mathrm{~mm} \times 30 \mathrm{~mm}$, used for preparation of fracture micrograph specimens) were applied as substrates. The sheets were prepared by preliminary grinding and mirror polishing, followed by ultrasonic cleaning in alcohol and acetone solution, and then were sputter-cleaned using Ar ion under $-950 \mathrm{~V}$ bias voltage to remove oxide and contaminant layer. 
An asymmetrical bipolar pulse DC magnetron sputtering (BPDMS) system was used to prepare the (CrAl)N and (CrAlSiY)N coatings. The two poles of a bipolar pulse power with frequency of $25 \mathrm{kHz}$, duty cycle of $80 \%$ were connected to $\mathrm{Cr}$ target and $\mathrm{Al}$ (or AlSiY) target respectively. The power of $\mathrm{Cr}$ or $\mathrm{Al}$ (or AlSiY) can be adjusted individually. Before deposition, $\mathrm{Cr}$ interlayer was deposited on the substrates for proper adhesion of the coatings. The deposition parameters were listed as following: base pressure $3 \times 10^{-3} \mathrm{~Pa}$, deposition temperature $260{ }^{\circ} \mathrm{C}$, total pressure of $\mathrm{N}_{2}$ and $\mathrm{Ar} 0.50 \mathrm{~Pa}, \mathrm{~N}_{2}$ partial pressure $0.40 \mathrm{~Pa}$, current of $\mathrm{Cr}$ target $4 \mathrm{~A}$, current of $\mathrm{Al}$ or AlSiY target $7 \mathrm{~A}$, pulsed bias $0 \mathrm{~V}$, $-100 \mathrm{~V},-200 \mathrm{~V}$ respectively, with frequency of $20 \mathrm{kHz}$ and duty cycle of $30 \%$, distance from target to substrates $15 \mathrm{~cm}$, deposition time $4.5 \mathrm{~h}$.

The surface and cross-sectional morphologies of the coatings were investigated using a Zeiss Ligma field emission scanning electron microscope (FESEM). The chemical composition of the deposited coatings was identified by EDS. The phase structures of the coatings were characterized by Bruker D8 ADVANCE X-ray diffractometer (XRD) with $\mathrm{Cu} \mathrm{K}_{\alpha}$ radiation. The hardness of coatings was tested by MH-6 Micro Hardness Tester (Everone Enterprises LTD.) with a load of 50 gf and 25 gf. The adhesive strength of coatings was evaluated by a WS-2005 Scratch Tester equipped with Rockwell C diamond tip (0.2 mm diameter).

\section{Results and Discussion}

The composition of (CrAl)N and (CrAlSiY)N of different pulse bias was listed in Table 1. The nitrogen content was about $52 \sim 54 \%$ and the ratio of $\mathrm{N} /(\mathrm{Cr}+\mathrm{Al})$ or $\mathrm{N} /(\mathrm{Cr}+\mathrm{Al}+\mathrm{Si}+\mathrm{Y})$ was close to 1:1 stoichiometry. For (CrAl)N coatings, the $\mathrm{Al}$ content decreased while the $\mathrm{Cr}$ content increased gradually with increased negative bias. Similarly, the content of $\mathrm{Al}, \mathrm{Si}$, and $\mathrm{Y}$ element decreased and $\mathrm{Cr}$ content increased in (CrAlSiY)N coating with improved negative bias. Under the bias-enhanced ion bombardment effect, the lighter $\mathrm{Al}$ and $\mathrm{Si}$ atoms and weak-bonded $\mathrm{Y}$ atoms are easier to being resputtered from coatings than the heavier $\mathrm{Cr}$ atom. Same regularity for (TiAl)N coatings was reported [12]. Under the same negative bias, the Cr content of $(\mathrm{CrAl}) \mathrm{N}$ coatings was lower than that of (CrAlSiY)N, indicating a lower sputtering rate of AlSiY target compared to that of Al target under the same deposition conditions.

Table 1. The composition of (CrAl)N and (CrAlSiY)N prepared under different pulse bias.

\begin{tabular}{lllllll}
\hline Substrate bias [V] & \multicolumn{3}{c}{ Composition [at.\%] } & & \multicolumn{2}{l}{ (Al+Si+Y)/(Cr+Al+Si+Y) } \\
& $\mathrm{Cr}$ & $\mathrm{Al}$ & $\mathrm{Si}$ & $\mathrm{Y}$ & $\mathrm{N}$ & \\
\hline (CrAl)N 0V & 26.76 & 19.02 & & & 54.22 & 41.5 \\
(CrAl)N 100V & 28.36 & 18.32 & & & 53.31 & 39.2 \\
(CrAl)N 200V & 29.26 & 17.13 & & & 53.61 & 36.9 \\
(CrAlSiY)N 0V & 30.94 & 14.85 & 1.44 & 0.77 & 52.70 & 33.0 \\
(CrAlSiY)N 100V & 31.09 & 13.31 & 0.86 & 0.40 & 54.34 & 31.9 \\
(CrAlSiY)N 200V & 32.06 & 12.48 & 0.64 & 0.32 & 54.50 & 29.5 \\
\hline
\end{tabular}

The XRD patterns of the as-deposited (CrAl)N and (CrAlSiY)N coatings were shown in Fig.1. Two kinds of coatings prepared under the same negative bias had the same phase structure of $\mathrm{B} 1 \mathrm{NaCl}$ and the similar preferred orientation. The pulse bias voltage exerted intensive influence on the preferred orientation for both kinds of the coatings. When no substrate bias was applied to the substrates, there existed strong (111) texture for both (CrAl)N and (CrAlSiY)N. While negative pulse bias voltage of $100 \mathrm{~V}$ or $200 \mathrm{~V}$ was put to use, (220) became the preferred orientation. In addition the peak broadening phenomenon appeared with the application of substrate bias. The average grain size was calculated from the width of the diffraction peak (220) using the well-known Scherrer's equation. 
Instrumental broadening and stress influence were not considered in this calculation. The average grain size of CrAlN deposited at $0 \mathrm{~V}$ was about $36 \mathrm{~nm}$, and that of (CrAlSiY)N was about $30 \mathrm{~nm}$. The application of the negative substrate bias decreased the grain size to 3-5 nm at the bias of $-100 \mathrm{~V}$ or $-200 \mathrm{~V}$ for $(\mathrm{CrAl}) \mathrm{N}$ and (CrAlSiY)N coatings.
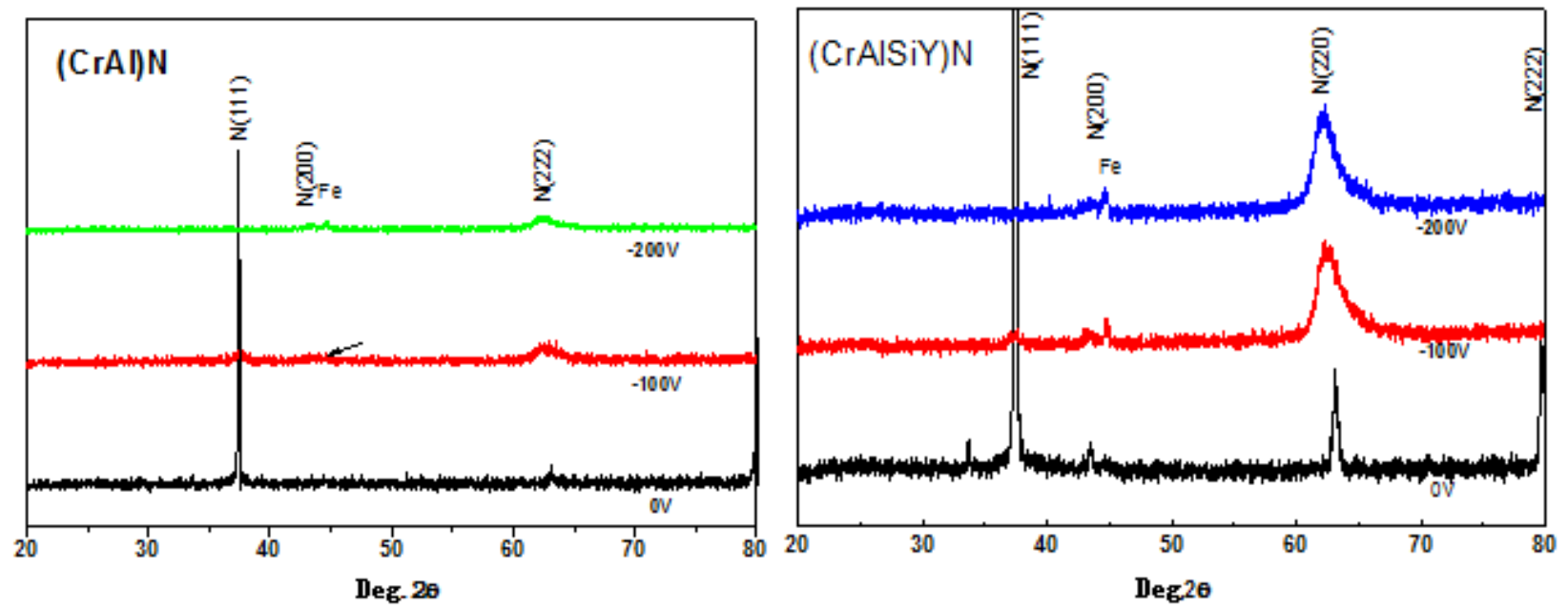

Fig. 1. XRD patterns of as-deposited (CrAl)N and (CrAlSiY)N coatings prepared under different bias.

The surface morphologies of $(\mathrm{CrAl}) \mathrm{N}$ and (CrAlSiY)N were shown in Fig.2. The surface appearance of (CrAlSiY)N was highly similar to that of $(\mathrm{CrAl}) \mathrm{N}$. The negative substrate bias strongly changed the surface morphologies. The surface of the coatings deposited at $0 \mathrm{~V}$ was composed of pebble-like particles with size up to $1000 \mathrm{~nm}$. The boundary lines of the particles could be identified clearly. Even there existed cracks along the boundaries. The size of the particles decreased to $400 \mathrm{~nm}$ when negative pulse bias voltage improved to $100 \mathrm{~V}$. While negative pulse bias voltage of $200 \mathrm{~V}$ was applied, no particles could be identified and the ridge topography appeared. Fig. 3 showed the cross-section of (CrAl)N and (CrAlSiY)N coatings deposited under various bias voltages. Without application of negative bias, typical columnar structure was formed. The average diameter of these columns was about $1000 \mathrm{~nm}$, which was the same as the size of the particles on the surface as mentioned above. So the particles should be the protrusions of the columnar structure. The coatings deposited under a negative bias voltage of $100 \mathrm{~V}$ showed a dense equiaxed-grain structure. After the negative pulse bias voltage reached up to $200 \mathrm{~V}$, a dense and featureless structure was gained. The increased substrate bias voltage greatly enhanced the ion bombardment effect, thus promoted the nucleation rate of the nitrides and inhabits the formation of columnar structure.
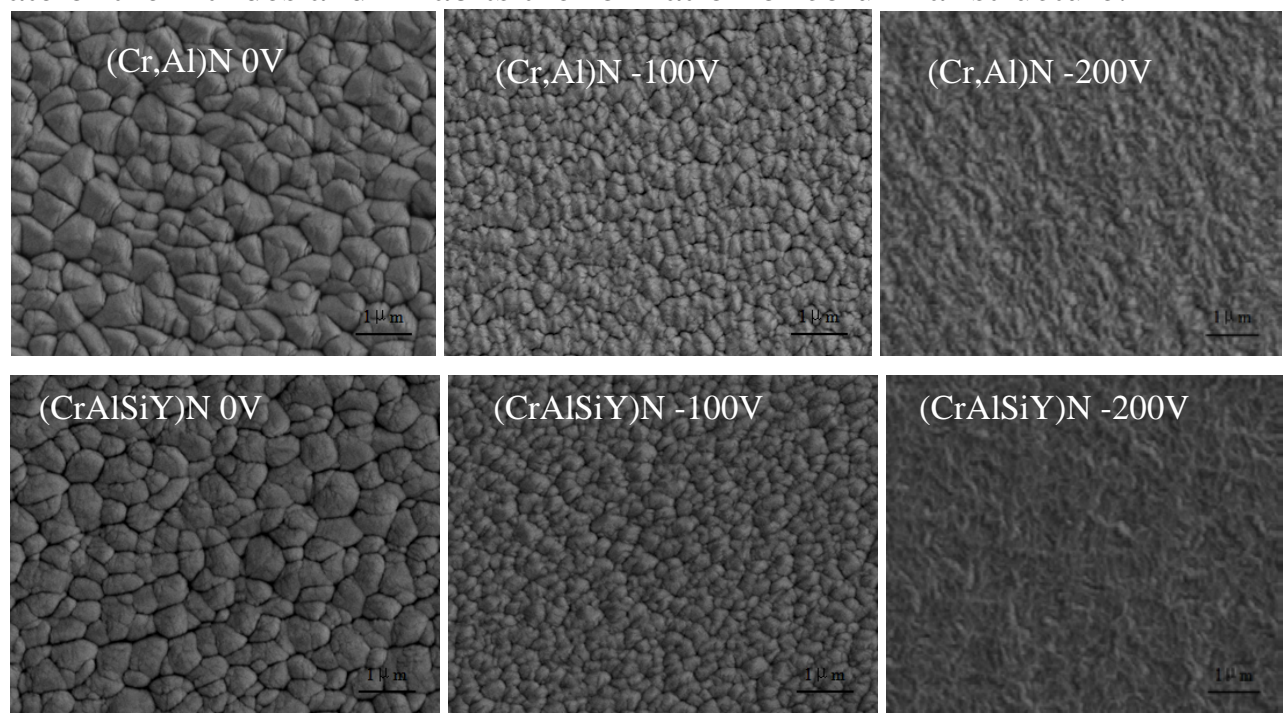

Fig. 2. Surface morphologies of (CrAl)N and (CrAlSiY)N coatings of different negative bias. 

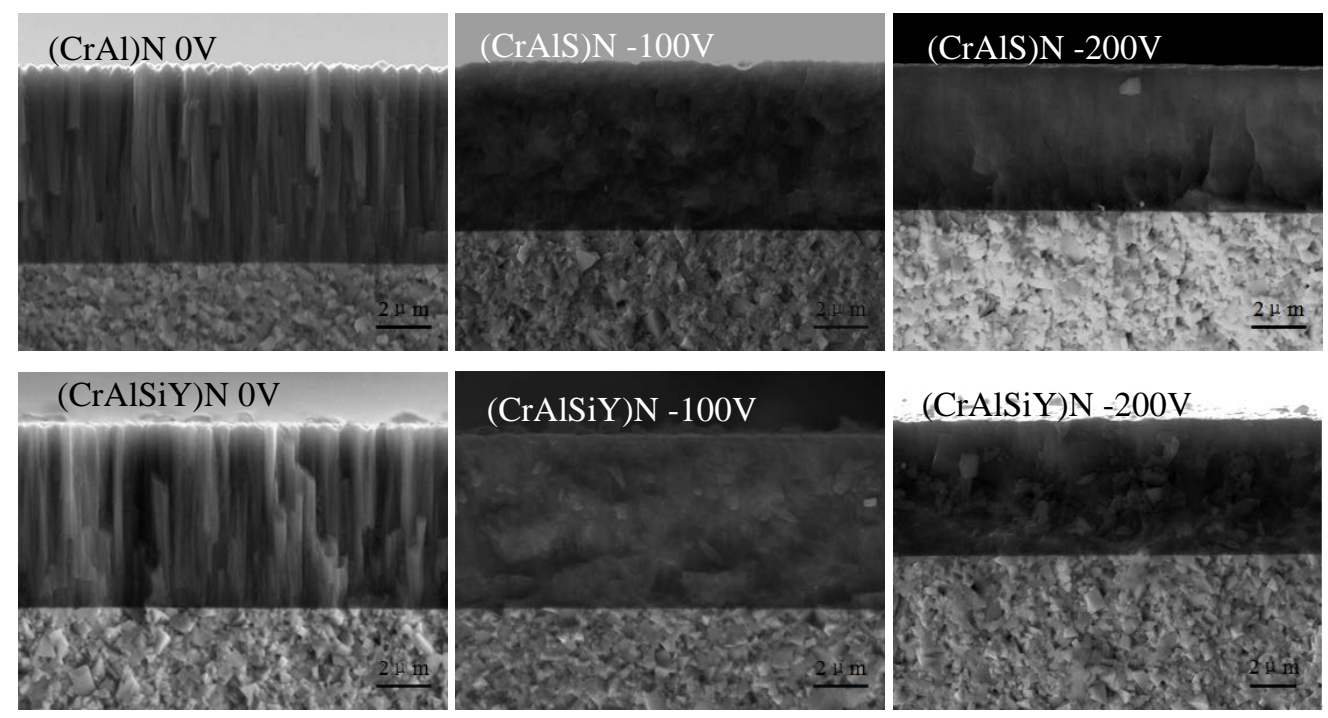

Fig. 3. Cross-section morphologies of (CrAl)N and (CrAlSiY)N coatings of different negative bias.

Vicker's hardness (HV) was tested by MH-6 Micro Hardness Tester with a load of 50 gf and 25 gf. The morphologies of the indentation were shown in Fig.5. The apparent hardness value of (CrAl)N of $0 \mathrm{~V},-100 \mathrm{~V}$ and $-200 \mathrm{~V}$ under the load of 50 gf was 5.02, 12.74 and $16.43 \mathrm{GPa}$ and that is 16.38, 31.31and 36.77 Gpa under the load of $25 \mathrm{gf}$. For (CrAlSiY)N of $0 \mathrm{~V},-100 \mathrm{~V}$ and $-200 \mathrm{~V}$, the apparent hardness value was 5.93, 16.06 and 18.66 Gpa under 50 gf load and 22.71, 41.96 and 44.47 Gpa under $25 \mathrm{gf}$ load. The hardness of steel substrate was about $2.8 \mathrm{GPa}$. While the thickness herein was about 4.5 7.5 $\mu \mathrm{m}$, so the gained apparent hardness was lower than the intrinsic hardness of the coatings owing to the influence of the soft steel substrate. And the bigger load was applied, the lower apparent hardness value was gained. The apparent harness of $25 \mathrm{gf}$ load was less influenced by the substrate compared with that of load of 50 gf. The micro hardness of (CrAl)N and (CrAlSiY)N coatings increased when negative bias increased from $0 \mathrm{~V}$ to $200 \mathrm{~V}$. Bombardment of energetic ions under high bias voltage on growing coatings led to compressive stress, fine grain and dense structure, which were in favor of improvement of the coating hardness. The addition of a small amount of Si and Y gave rise to a considerable increase of hardness for the coatings. As mentioned above, $(\mathrm{CrAl}) \mathrm{N}$ and (CrAlSiY)N prepared under the same bias possessed the same phase composition and similar micro structure. But the (CrAlSiY)N coatings exhibited higher hardness. The amount of Si is so small that it was impossible to form $\mathrm{Si}_{3} \mathrm{~N}_{4}$. The increase of the hardness should be ascribed to the solid solution strengthening and the grain strengthening.

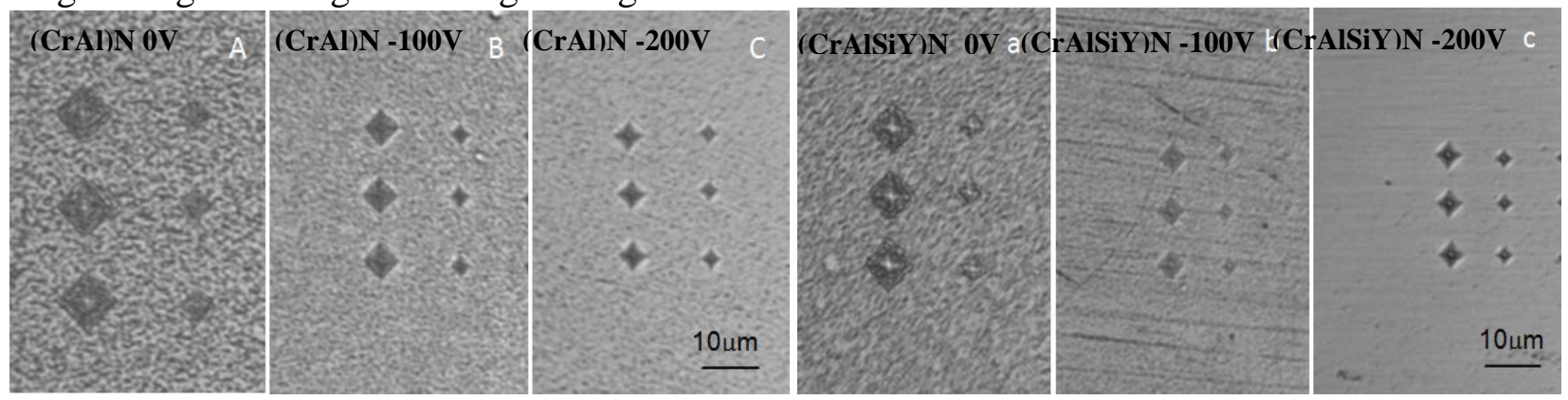

Fig. 4. Morphologies of indentation under load of $25 \mathrm{gf}$ and $50 \mathrm{gf}$ on the surface of (CrAl)N and (CrAlSiY)N coatings.

Fig.5 showed the scratch images and the corresponding acoustic emission (AE) signals profiles for (CrAl)N and (CrAlSiY)N coatings deposited under different negative bias. The test system was composed of a hard coating and a soft substrate. During the loading process, the scratch depth and width increased with an increasing load. The minor signals were from deformation and surface friction. The next main signal was regarded as the marks of the adhesive failure of the coating and the 
corresponding load was recorded as critical load (Lc). The (Lc) markedly dropped with increase of negative bias voltage for $(\mathrm{CrAl}) \mathrm{N}$ and $(\mathrm{CrAlSiY}) \mathrm{N}$ coatings. (CrAlSiY)N coatings showed lower critical load than $(\mathrm{CrAl}) \mathrm{N}$ coatings. When negative bias increased or element of $\mathrm{Si}$ and $\mathrm{Y}$ were introduced, the part delamination occurred sooner during the process of scratch, which indicated a decreasing toughness due to increasing negative bias and addition of Si and Y. It was mentioned above that the higher bias voltage and the addition of Si and Y led to the harder and thinner coatings. They were no easy to coordinating to the deformation of the steel substrate and showed lower Lc. For the system of a hard coating on a soft substrate, the Lc could not reflect the actual adhesive strength. It can be identified there was no continuous spallation within the $50 \mathrm{gf}$ load for all the coatings.
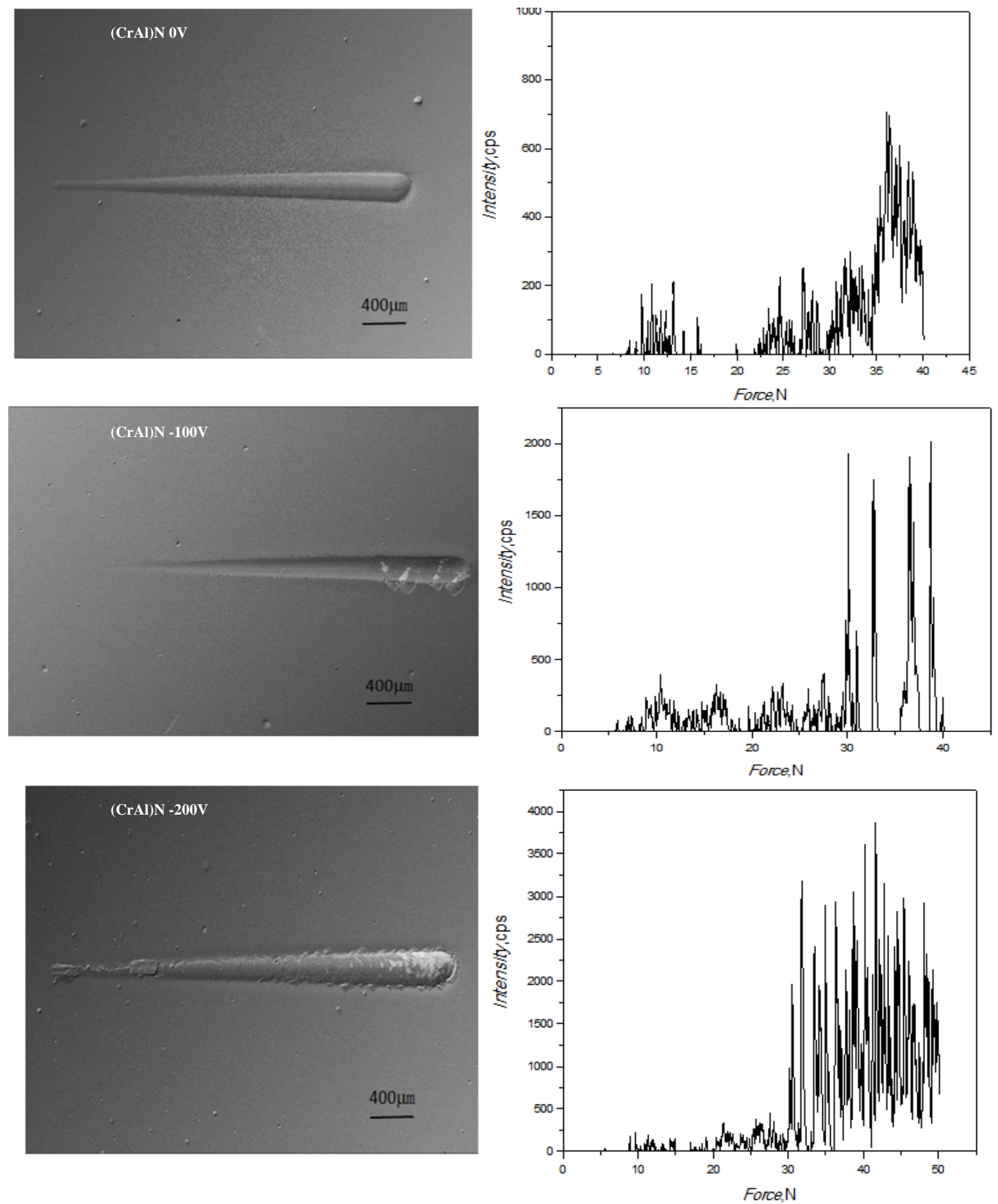

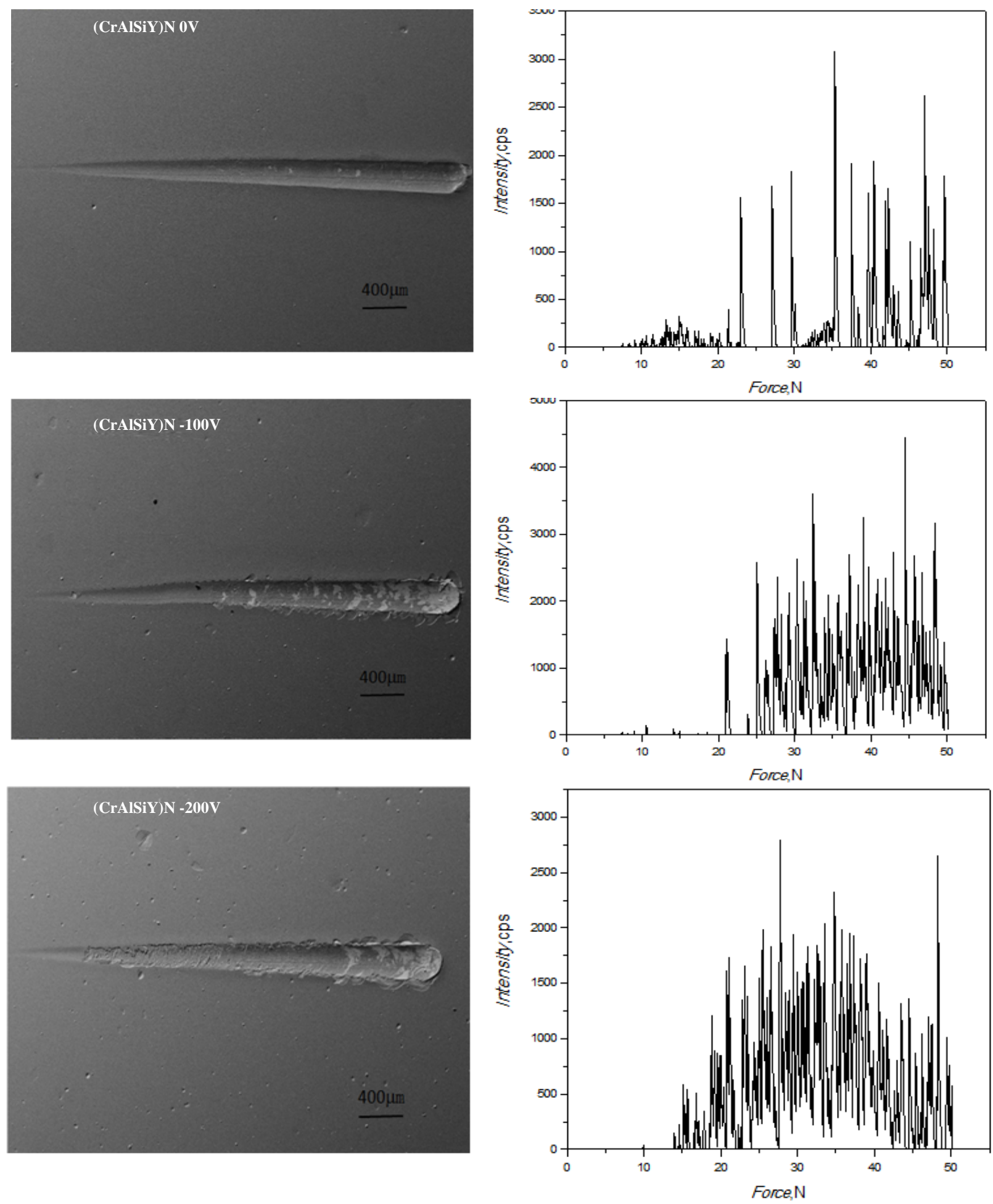

Fig. 5. Scratch morphologies and the corresponding AEsignals for (CrAl)N and (CrAlSiY)N coatings of different bias.

\section{Summary}

(CrAl)N and (CrAlSiY)N had the same phase composition and similar micro structure prepared under the same negative bias. The incorporation of Si and $\mathrm{Y}$ into the coatings did not give rise to the obvious change of phase structure and texture orientation, but led the notable increase of hardness and decrease of scratch critical load. With the increasing negative bias, the structure density improved and the hardness increased remarkably. 


\section{Acknowledgement}

The project supported by the National Natural Science Foundation of China (Grant No. 51461016), the Province Natural Science Foundation of Jiangxi (Grant No.20122BAB206019) and the Provincial Scientific and Technological Support Plan of Jiangxi (Grant No. 20122BBE500030).

\section{References}

[1] Y.L. Su, S.H. Yao, On the performance and application of CrN coating, Wear 205(1997) 112-119.

[2] D.B. Lee,Y.C. Lee, S.C. Kwon, High temperature oxidation of a CrN coating deposited on a steel substrate by ion plating, Surface and Coatings Technology 141 (2001) 227-231.

[3] Shicai Yang, Kevin Cooke, Hailin Sun, Xiaoying Li, Kaijie Lin, Hanshan Dong, Development of advanced duplex surface systems by combining CrAlN multilayer coatings with plasma nitrided steel substrates, Surface and Coatings Technology 236 (2013) 2-7.

[4] Y.C. Chim, X.Z. Ding, X.T. Zeng, S. Zhang, Oxidation resistance of TiN, CrN, TiAlN and CrAlN coatings deposited by lateral rotating cathode arc,Thin Solid Films 517(2009) 4845-4849

[5] S. Khamseh, M. Nose, T. Kawabata, K. Matsuda, S. Ikeno, Oxidation Resistance of CrAlN Films with Different Microstructures Prepared by Pulsed DC Balanced Magnetron Sputtering System, Material Transactions 51 (2010) 2 271-276.

[6] Chun-Chi Chang, Hsien-Wei Chen, Jyh-Wei Lee, Jenq-Gong Duha, Influence of Si contents on tribological characteristics of CrAlSiN nanocomposite coatings, Thin Solid Films 584 (2015) 46-51.

[7] Dong Bok Lee, Thuan Dinh Nguyen, Sun Kyu Kim, Air-oxidation of nano-multilayered CrAlSiN thin films between 800 and $1000{ }^{\circ} \mathrm{C}$, Surface and Coatings Technology 203 (2009) 1199-1204.

[8] Z.B. Qi, Z.T. Wu, Z.C. Wang, Improved hardness and oxidation resistance for CrAlN hard coatings with Y addition by magnetron co-sputtering, Surface and Coatings Technology 259 (2014,) 146-151.

[9] S. Mato, G. Alcalá, M. Brizuela, R. Escobar Galindo, F.J. Pérez, J.C. Sánchez-López, Long-term high temperature oxidation of $\mathrm{CrAl}(\mathrm{Y}) \mathrm{N}$ coatings in steam atmosphere, Corrosion Science 80 (2014) 453-460.

[10]Kun-Ming Chen, Deng-An Tsai, Hao-Chia Liao, In-Gann Chen, Weng-Sing Hwang, Investigation of Al-Cr alloy targets sintered by various powder metallurgy methods and their particle generation behaviors in sputtering process, Journal of Alloys and Compounds 663 (2016) 52-59.

[11]Mingsheng Li, Yongzhong Fan, Shujuan Zhang and Changjie Feng, Structure and Property of CrAlN Coatings prepared by bipolar pulsed dual magnetron sputtering(BPDMS) under different pulse bias voltage, advanced materials research, 189-193 (2011) 668-671.

[12] Mingsheng Li, Fuhui Wang, Effects of nitrogen partial pressure and pulse bias voltage on (Ti,Al)N coatings by arc ion plating ,Surface and Coatings Technology 167 (2003) 197-202 . 